\title{
A Design-oriented Charge-based Simplified Model for FDSOI MOSFETs
}

\author{
Alessandro Pezzotta*, Farzan Jazaeri*, Heorhii Bohuslavskyi ${ }^{\dagger}$, Louis Hutin ${ }^{\dagger}$, Christian Enz $^{*}$ \\ ${ }^{*}$ ICLAB, École Polytechnique Fédérale de Lausanne (EPFL), Neuchâtel, Switzerland; ${ }^{\dagger}$ CEA Leti, Grenoble, France \\ Email: alessandro.pezzotta@epfl.ch
}

\begin{abstract}
In this paper a design-oriented model for asymmetrical double-gate (ADG) MOSFETs is proposed. Including the back-gate effect into the original simplified EKV bulk model requires only one additional parameter to the existing four, and extends the simplified EKV model to FDSOI processes. This will help the designer to find the right trade-off in terms of design parameters, including the back-gate biasing. A comparison with measurement results from a 28-nm FDSOI CMOS process is provided, assessing the excellent accuracy of the proposed.

Index Terms-simplified EKV, modeling, FDSOI, back-gate
\end{abstract}

\section{INTRODUCTION}

In most of the applications that nowadays are dealing with integrated circuits, low-power operation is a key aspect. Due to this fact, conventional design methodologies have been revised in order to exploit at best all the features of advanced technologies [1]. In that sense, the simplified EKV model (sEKV) [2], [3], based on the inversion coefficient $(I C)$, has demonstrated its effectiveness in describing the performance of MOSFETs in all inversion conditions while using only a few parameters. The key of this capability, together with the charge-related basis, is the normalization, that strips off the dependence to a specific technology which is then captured by only a few parameters. This makes the simplified EKV model suitable also as a benchmark for technologies, in addition to helping the designer to explore the design space and find the right trade-off in terms of design parameters [2].

However, the technologies portfolio has been growing rapidly as the scaling-down process gets close to reach physical limits achievable with a standard bulk planar technology. In the latest years, two main alternatives to bulk process has emerged, i.e. finFET and FDSOI processes [4], [5]. If in the case of finFET, the sEKV bulk model is still suitable [2], for FDSOI back-gate effects have to be considered. Therefore, this further degree of freedom has been addressed through a re-adaptation of the sEKV model, so that the design flow can directly focus on the performance trade-offs linked also to the back-gate biasing.

The paper is organized as follows. In Section II, the sEKV model is briefly recalled and its extension for ADG processes is detailed, followed by the parameter extraction flow described in Section III. Its validation with respect to measurement results from a 28-nm FDSOI CMOS process is shown in Section IV, while the practical implications of the proposed approach are highlighted in Section V. At the end, conclusions are drawn.

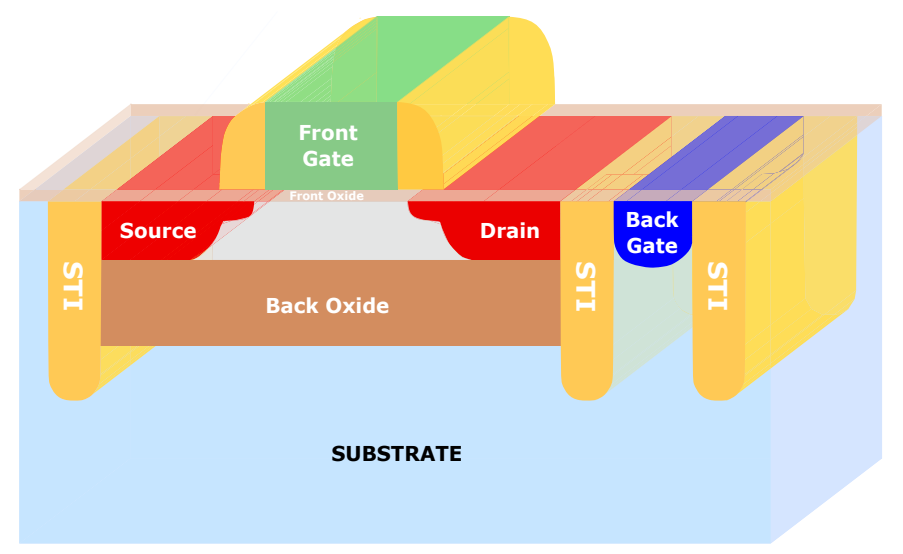

Fig. 1: Sketch of a typical FDSOI MOSFET.

\section{MODEL DESCRIPTION}

As a starting point, the simplified EKV model is considered [2]. In order to recall it, its derivation basics are cited below. The net normalized drain current that flows into the device can be expressed as a function of the normalized source and drain charges, $q_{\mathrm{s}}$ and $q_{\mathrm{d}}$ respectively, as $i_{\mathrm{d}}=q_{\mathrm{s}}+q_{\mathrm{s}}^{2}-q_{\mathrm{d}}-q_{\mathrm{d}}^{2}$.

The first simplification introduced assumes that the device is operating in saturation region, that is actually the case in most of its practical uses in analog design. In this conditions, $q_{\mathrm{d}}$ can be reasonably neglected, yielding:

$$
I C=\frac{\left.I_{\mathrm{D}}\right|_{\text {saturation }}}{I_{\mathrm{spec}}}=i_{\mathrm{dsat}}=q_{\mathrm{s}}+q_{\mathrm{s}}^{2},
$$

where the Inversion Coefficient $I C$ is defined as the drain current in saturation normalized with respect to the specific current $I_{\text {spec }}$.

Nevertheless, in ultra-scaled technologies, Short-Channel Effects (SCEs) play a crucial role when the channel length $L$ approaches the minimum feature size. When this occurs, the simple relation in (1) is not effective. In order to take into account SCEs, and in particular Velocity Saturation (VS), the following relation is considered instead [6]:

$$
I C=\frac{4\left(q_{\mathrm{s}}+q_{\mathrm{s}}^{2}\right)}{2+\lambda_{\mathrm{c}}+\sqrt{4\left(1+\lambda_{\mathrm{c}}\right)+\lambda_{\mathrm{c}}^{2}\left(1+2 q_{\mathrm{s}}\right)^{2}}},
$$

where $\lambda_{\mathrm{c}}=L_{\mathrm{sat}} / L$ is the VS parameter, corresponding to the relative portion of channel in full velocity saturation. 
In order to make a link between the drain current and the gate voltage, and hence have an analytical expression for the MOSFET's transfer characteristic, the EKV model is again exploited. The latter provides a charge-to-voltage relation expressed as

$$
2 q_{\mathrm{s}}+\ln q_{\mathrm{s}}=v_{\mathrm{p}}-v_{\mathrm{s}},
$$

where $v_{\mathrm{p}}$ and $v_{\mathrm{s}}$ are the normalized pinch-off and source voltage respectively. Notice that all voltages are normalized to the thermal voltage $U_{\mathrm{T}}=k T / q$, where $k$ is the Boltzmann constant, $T$ is the temperature and $q$ is the electron charge.

The crucial statement that enables the inclusion of backgate effect is the re-definition of the channel pinch-off voltage $v_{\mathrm{p}}$. The sEKV model for bulk processes defines it as $\left(v_{\mathrm{g}}\right.$ $\left.v_{\mathrm{t} 0}\right) / n$, that is the difference between the gate voltage and the threshold voltage, rescaled with respect to the slope factor $n$. Targeting ADG processes, this definition has to be modified accounting for the two independent gates, namely the frontgate voltage $v_{\text {fg }}$ and the back-gate voltage $v_{\text {bg }}$.

In the range of voltage useful for the designer, the impact of the back-gate voltage can simply be modeled as a threshold voltage with a linear dependence to $v_{\mathrm{bg}}$ (this claim will be confirmed in Section IV), so the pinch-off voltage $v_{\mathrm{p}}$ can be defined as

$$
v_{\mathrm{p}}=\frac{v_{\mathrm{fg}}-v_{\mathrm{t}}}{n}=\frac{v_{\mathrm{fg}}-\left(v_{\mathrm{t} 0}-k_{\mathrm{ox}} v_{\mathrm{bg}}\right)}{n},
$$

where $v_{\mathrm{t} 0}$ is considered as the threshold voltage when $v_{\mathrm{bg}}$ is set to 0 , and $k_{\mathrm{Ox}}$ is a constant parameter directly linked to the device structure, namely to the the two gate oxide capacitances ratio:

$$
k_{\mathrm{ox}}=\frac{C_{\mathrm{ox}_{\mathrm{b}}}}{C_{\mathrm{ox}_{\mathrm{f}}}}=\frac{E O T_{\mathrm{f}}}{E O T_{\mathrm{b}}} .
$$

Hence, inserting Eqs. (2) and (4) into (3), the voltage-current expression related to the front- and back-gate voltages can be derived:

$$
\begin{array}{r}
\frac{v_{\mathrm{fg}}-\left(v_{\mathrm{t} 0}-k_{\mathrm{ox}} v_{\mathrm{bg}}\right)}{n}-v_{\mathrm{s}}=\sqrt{\left(1+\lambda_{\mathrm{c}} I C\right)^{2}+4 I C} \\
+\ln \left[\sqrt{\left(1+\lambda_{\mathrm{c}} I C\right)^{2}+4 I C}-1\right]-(1+\ln 2) .
\end{array}
$$

It is worth to make some remarks. First of all, notice that (6) expresses the front-gate voltage as a function of $I C$, and it is not invertible. Secondly, for the drain current normalization, the $I_{\mathrm{spec}}$ is given by $I_{\mathrm{spec}_{\square}} \frac{W}{L}$, where $I_{\mathrm{spec}_{\square}}=2 n \mu_{0} C_{\mathrm{ox}} U_{\mathrm{T}}^{2}$. Since for these structures the analytical individual definition of the low-field mobility $\mu_{0}$ and $C_{\mathrm{ox}}$ is not trivial, and the actual goal of this model is to provide an instrument for earlystage design procedures rather than a physics-based compact model, the proposed approach is to replace their product with a generic parameter $\beta$, extracted through data fitting and only related to the specific process.

\section{PARAmeter EXtraction Flow}

As a whole, the parameters included into sEKV are now five. Together with the four already included into the sEKV for bulk, namely $n, I_{\mathrm{spec}_{\square}}, L_{\mathrm{sat}}$ and $v_{\mathrm{t} 0}, k_{\mathrm{ox}}$ is considering the back-gate impact on the threshold voltage.

The first necessary step for extracting these parameters regards the standard extraction flow for $n, I_{\text {spec }_{\square}}, L_{\text {sat }}$ and $v_{\mathrm{t} 0}$, to be performed as described in [2] and synthesized in the flow diagram in Fig. 2, starting from long-channel towards short-channel devices. All this has to be performed initially at $V_{\mathrm{bg}}=0 \mathrm{~V}$, then also for several back-gate bias points, positive and negative.

Notice that the parameter database consistency among all the lengths has to be constantly checked. Namely, $I_{\text {spec }_{\square}}$ variation versus $L$ can be justified only in case of a corresponding variation in $n$, while $L_{\text {sat }}$ should remain constant. Thus, once verified that the parameter set is consistent, and eventually performed a general fine-tuning, the $k_{\mathrm{ox}}$ parameter can be extracted.

Initially, the threshold voltage at $V_{\mathrm{bg}}=0 \mathrm{~V}$, normalized with respect to $U_{\mathrm{T}}$, will assume the value of $v_{\mathrm{t} 0}$. Then, considering all the threshold voltage values extracted from

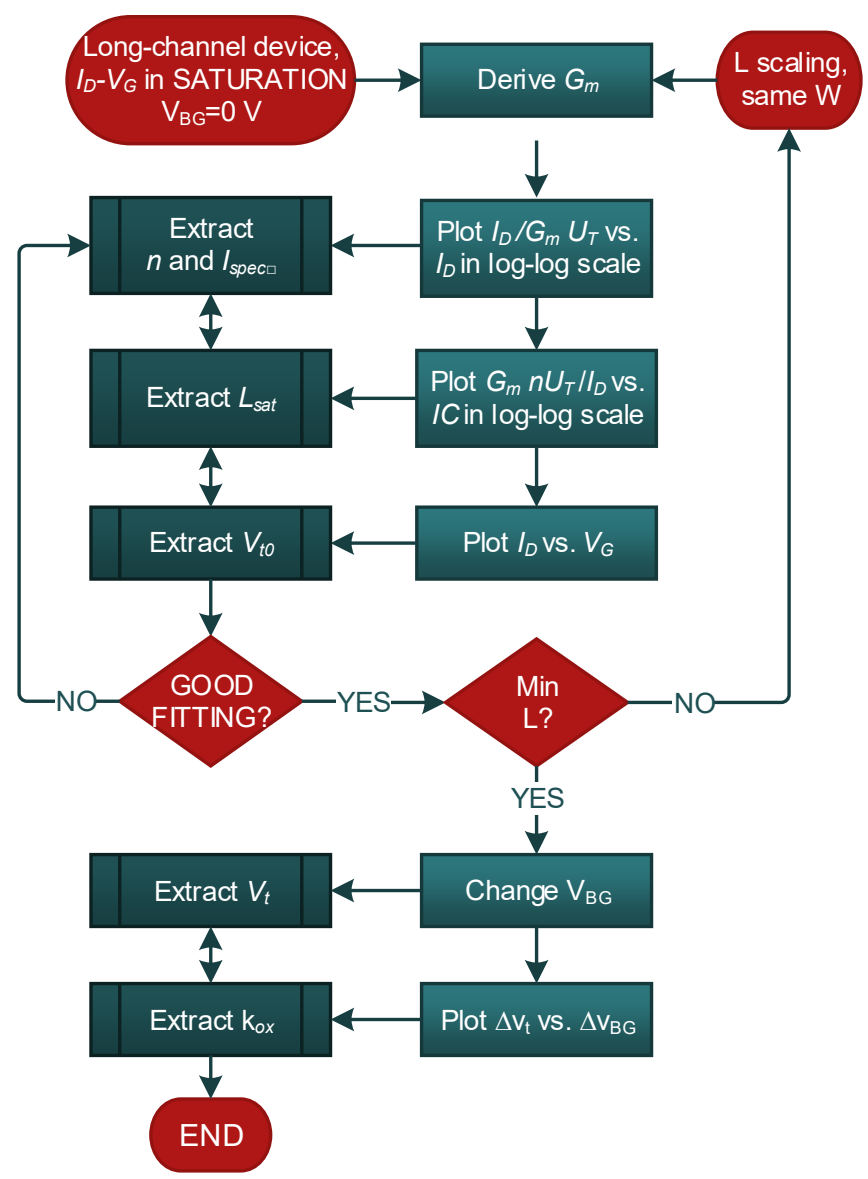

Fig. 2: Simplified EKV parameters extraction flow. 


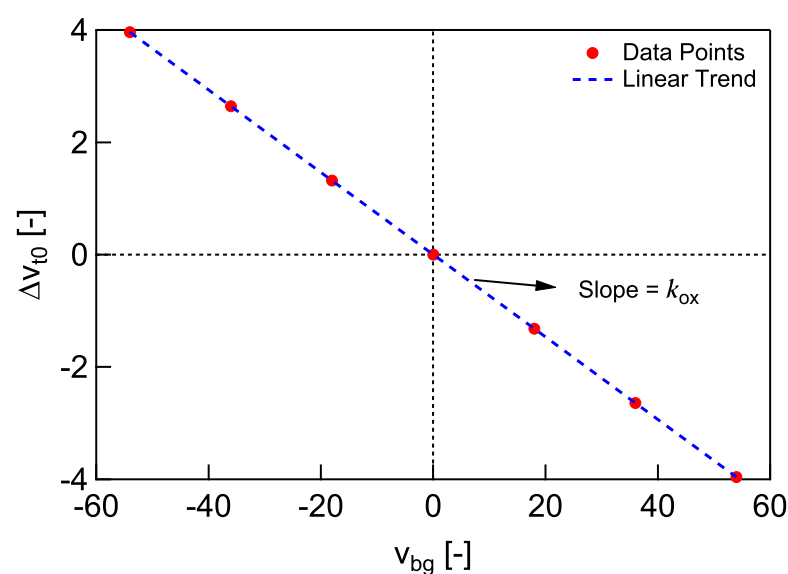

Fig. 3: $\Delta v_{\mathrm{t} 0}$ vs. $v_{\mathrm{bg}}$ plot used for $k_{\mathrm{ox}}$ extraction.

others back-gate bias points and for a specific channel length, it is possible to plot the relative variation of the threshold voltage, i.e. $\Delta v_{\mathrm{t} 0}$, with respect to $v_{\mathrm{bg}}$, as shown in Fig. 3 . Specifically, the collection of obtained points should follow a linear trend with zero intercept, whose slope is $k_{\mathrm{ox}}$ indeed. Notice that the value of $k_{\mathrm{ox}}$ should not depend on $L$, meaning that for each channel length analyzed, the extracted $k_{\mathrm{ox}}$ should be identical.

\section{Model VALIDATION}

In order to confirm the validity of the previously described sEKV model, a set of measurement data from a 28-nm FDSOI technology is considered (Figs. 4 to 6). In detail, two process options with different front gate oxide thicknesses have been analyzed, whose reference is set to "GO1" and "GO2".

Since the two families of devices differ only by their equivalent front-gate oxide thickness $E O T_{\mathrm{f}}$, by means of the obtained values for $k_{\mathrm{ox}}$ in the two cases the equivalent backoxide thickness $E O T_{\mathrm{b}}$ can be extracted. If the theory is valid, the $E O T_{\mathrm{b}}$ values extracted from both families should match. The dataset details are listed in Table I.

TABLE I: 28-nm FDSOI dataset specifications.

\begin{tabular}{|c|c|c|}
\hline & G01 & GO2 \\
\hline Type & $\begin{array}{ll}\text { NMOS } & \text { PMOS }\end{array}$ & $\begin{array}{ll}\text { NMOS } & \text { PMOS }\end{array}$ \\
\hline TOXE & $1.55 \mathrm{~nm}$ & $3.7 \mathrm{~nm}$ \\
\hline$\overline{\mathrm{W}}$ & $1 \mu \mathrm{m}$ & $2 \mu \mathrm{m}$ \\
\hline $\mathrm{L}$ & $\begin{array}{c}1 \mu \mathrm{m} \\
28 \mathrm{~nm}\end{array}$ & $2 \mu \mathrm{m}$ \\
\hline
\end{tabular}

Following the flow described in Section III and considering the case of GO1 Thin-Oxide nMOSFETs (Figs. 4a to 4c), the extracted $k_{\text {ox }}$ value is $66.7 \mathrm{mV} / \mathrm{V}$. Since the $E O T_{\mathrm{f}}$ for this family is equal to $1.55 \mathrm{~nm}$, from (5) the value obtained for $E O T_{\mathrm{b}}$ is $23.24 \mathrm{~nm}$.

As a remark, the specific FDSOI process considered is fabricated using the same back-oxide thickness both for GO1 and $\mathrm{GO} 2$ devices. Thus, in order to have the model assessed, from the obtained $E O T_{\mathrm{b}}$ value and the $k_{\mathrm{ox}}$ extracted as in Fig. $6 c$ for GO2 Thick-Oxide nMOSFET $(158 \mathrm{mV} / \mathrm{V})$, the
$E O T_{\mathrm{f}}$ indicated in Table I should be obtained. Indeed, using again (5), a value of $3.67 \mathrm{~nm}$ for $E O T_{\mathrm{f}}$ is derived, with less than $1 \%$ discrepancy.

Proceeding the same way for Thin-Oxide pMOSFETs (Figs. $5 \mathrm{a}$ to $5 \mathrm{c}, E O T_{\mathrm{f}}=1.7 \mathrm{~nm}$ ), the extracted $k_{\text {ox }}$ results in $73.3 \mathrm{mV} / \mathrm{V}$, that gives an $E O T_{\mathrm{b}}$ of $23.28 \mathrm{~nm}$. Consequently, for the Thick-Oxide family an $E O T_{\mathrm{f}}$ of $3.68 \mathrm{~nm}$ is obtained, close to the expected value of $3.7 \mathrm{~nm}$.

Notice that for the same device type and family, a consistent set of sEKV parameters has been extracted. This may lead to a non-ideal fit in some of the curves shown in Figs. 4 to 6 (ex.: moderate inversion region of Fig. 5b). However, as already reminded, the goal of the approach is to provide an instrument for early-stage design procedures rather than a physics-based compact model.

\section{Practical consequences}

Parameter $k_{\text {ox }}$ changes the effective threshold voltage with respect to the back-gate voltage and hence shifts the I-V curves to the left of the $v_{\mathrm{bg}}=0$ curve when $v_{\mathrm{bg}}$ is positive or to the right when $v_{\mathrm{bg}}$ is negative. The larger $k_{\mathrm{ox}}$, e.g. the larger the ratio of oxide thicknesses, the larger the spread of the curve for an equal $v_{\text {bg }}$ step.

Considering the process, it is evident from (5) that the back-gate effect is weaker when the back-oxide is considerably thicker than the front-oxide, as in GO1 process family. However, this effect can be boosted as far as the two oxides thicknesses get similar, which is the case of $\mathrm{GO} 2$ process family.

From a design perspective, the parameter $k_{\text {ox }}$ can be exploited as a new degree-of-freedom. Indeed, it represents the direct measure of the possible shift in the range of $I C$ values available for a specific geometry and front-gate bias point. This can eventually be employed into a quantitative design of dynamic non-idealities compensation circuits, which can actively counteract on offset/mismatch phenomena that affect a specific structure [7].

\section{Conclusions}

A charge-based simplified model for ADG processes is proposed, that is able to include the effect of back-gate biasing on the threshold voltage by means of only one additional parameter, $k_{\mathrm{ox}}$, with respect to the four used in the bulk model. Through measurement data analysis on two device families from a 28-nm FDSOI process, the approach has demonstrated to be effective, by checking the correspondence between extracted physical parameters, i.e. the equivalent front- and back-oxide thicknesses, and their expected value.

The straightforward consequence of this approach, is that even for FDSOI processes, the $I C$-based design methodology [8] remains valid including the additional back-gate voltage. On top of that, the new parameter $k_{\mathrm{ox}}$ can act as a range shifter for the charge-to-voltage relationship, meaning that for the same front-gate bias point and geometry, the device can operate in a range of $I C \mathrm{~s}$, directly linked to the value of $k_{\mathrm{ox}}$. This has been already shown practically [5], but now it can be assessed quantitatively and, above all, simply. 


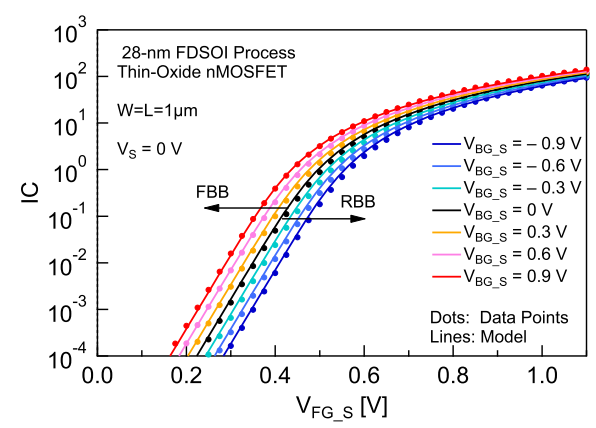

(a)

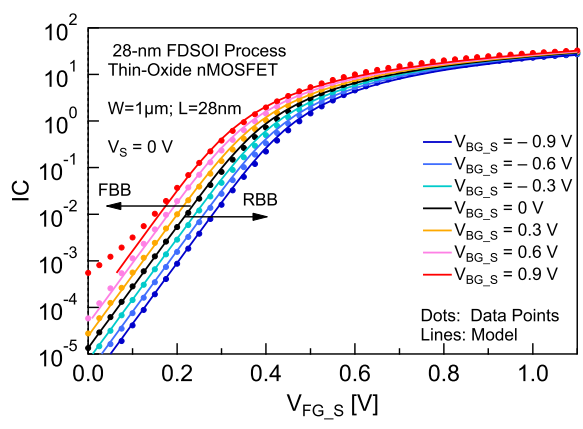

(b)

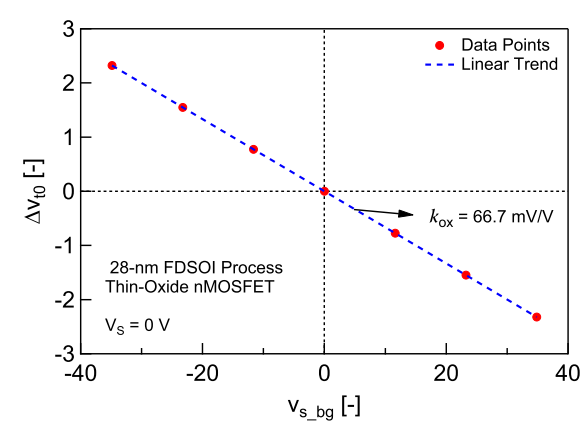

(c)

Fig. 4: a) IC vs front-gate voltage for Long-Channel Thin-Oxide nMOSFET (GO1) at different back-gate bias points. b) IC vs front-gate voltage for ShortChannel Thin-Oxide nMOSFET (GO1) at different back-gate bias points. c) $k_{\mathrm{ox}}$ extraction from $\Delta v_{\mathrm{t} 0}$ vs $v_{\mathrm{bg}}$ plot for Thin-Oxide nMOSFET (GO1).

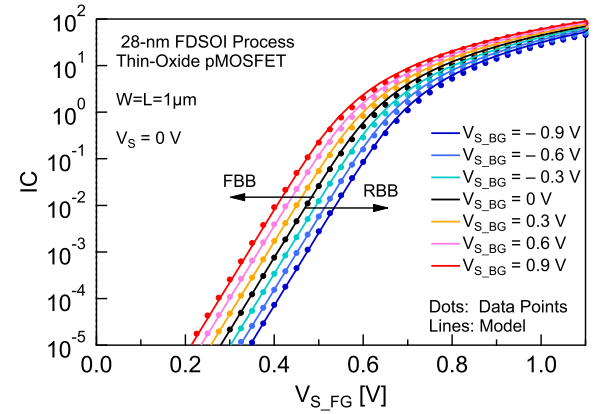

(a)

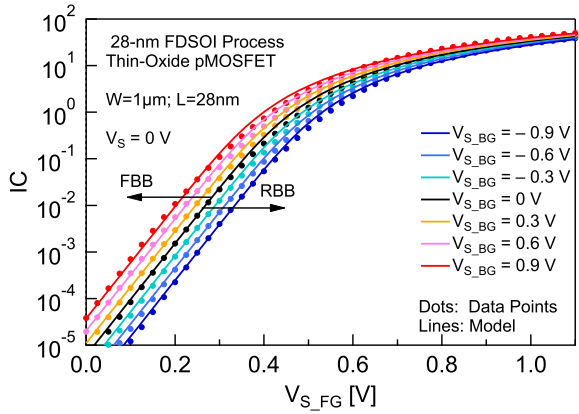

(b)

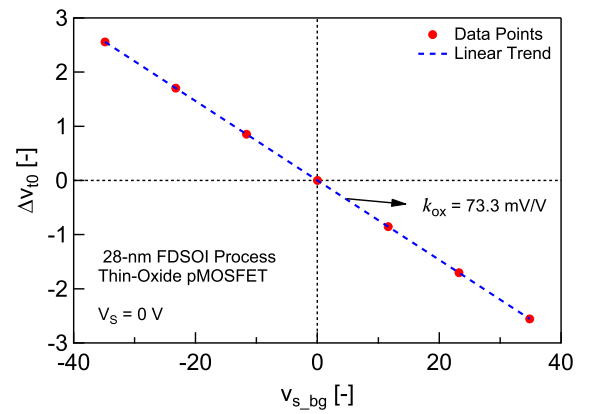

(c)

Fig. 5: a) IC vs front-gate voltage for Long-Channel Thin-Oxide pMOSFET (GO1) at different back-gate bias points. b) IC vs front-gate voltage for ShortChannel Thin-Oxide pMOSFET (GO1) at different back-gate bias points. c) $k_{\mathrm{ox}}$ extraction from $\Delta v_{\mathrm{t} 0}$ vs $v_{\mathrm{bg}}$ plot for Thin-Oxide pMOSFET (GO1).

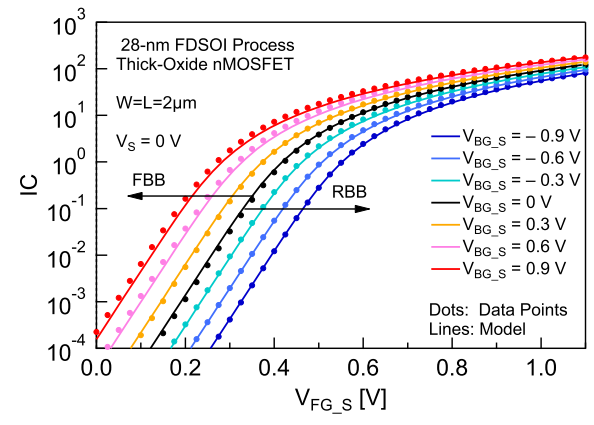

(a)

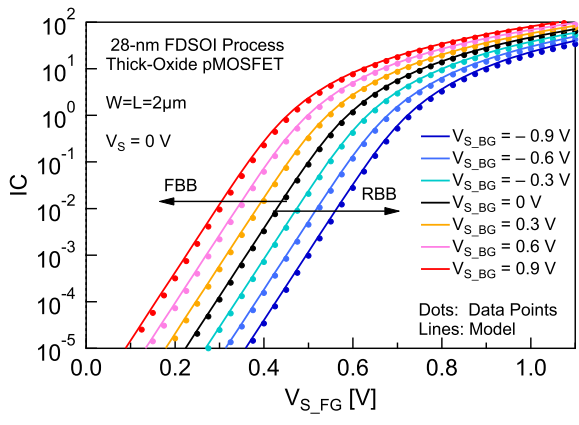

(b)

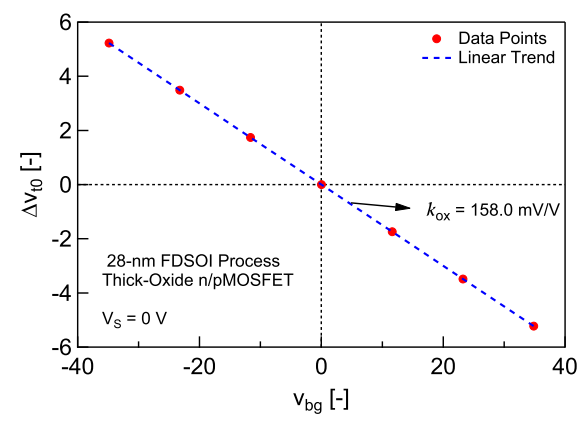

(c)

Fig. 6: a) IC vs front-gate voltage for Long-Channel Thick-Oxide nMOSFET (GO2) at different back-gate bias points. b) IC vs front-gate voltage for Long-Channel Thick-Oxide pMOSFET (GO1) at different back-gate bias points. c) $k_{\mathrm{ox}}$ extraction from $\Delta v_{\mathrm{t} 0}$ vs $v_{\mathrm{bg}}$ plot for Thick-Oxide n/pMOSFET (GO2).

\section{REFERENCES}

[1] W. Sansen, "Minimum Power in Analog Amplifying Blocks: Presenting a Design Procedure," IEEE Solid-State Circuits Magazine, vol. 7, no. 4 pp. 83-89, Fall 2015.

[2] C. Enz, F. Chicco, and A. Pezzotta, "Nanoscale MOSFET Modeling: Part 1: The Simplified EKV Model for the Design of Low-Power Analog Circuits," IEEE Solid-State Circuits Magazine, vol. 9, no. 3, pp. 26-35, Summer 2017.

[3] C. C. Enz and E. A. Vittoz, Charge-Based MOS Transistor Modeling The EKV Model for Low-Power and RF IC Design. John Wiley, 2006.

[4] D. Hisamoto et al., "FinFET-a self-aligned double-gate MOSFET scalable to $20 \mathrm{~nm}$," IEEE Transactions on Electron Devices, vol. 47, no. 12, pp. 2320-2325, 2000
[5] A. Cathelin, "Fully Depleted Silicon on Insulator Devices CMOS: The 28-nm Node Is the Perfect Technology for Analog, RF, mmW, and MixedSignal System-on-Chip Integration," IEEE Solid-State Circuits Magazine, vol. 9, no. 4, pp. 18-26, Fall 2017.

[6] A. Mangla et al., "Design Methodology for Ultra Low-power Analog Circuits Using Next Generation BSIM6 MOSFET Compact Model,' Microelectronics Journal, vol. 44, no. 7, pp. 570-575, July 2013.

[7] M. Raj, S. Saeedi, and A. Emami, "A Wideband Injection Locked Quadrature Clock Generation and Distribution Technique for an EnergyProportional 16-32 Gb/s Optical Receiver in 28 nm FDSOI CMOS," IEEE Journal of Solid-State Circuits, vol. 51, no. 10, pp. 2446-2462, Oct 2016.

[8] C. Enz, F. Chicco, and A. Pezzotta, "Nanoscale MOSFET Modeling. Part 2: Using the Inversion Coefficient as the Primary Design Parameter," IEEE Solid-State Circuits Magazine, vol. 9, no. 4, pp. 73-81, Fall 2017. 